\title{
A retrospective study of immune checkpoint inhibitor-associated myocarditis in a single center in China
}

\author{
Feng Wang ${ }^{1,2 \#}$, Xinchen Sun ${ }^{1 \#}$, Shukui Qin ${ }^{2}$, Haiqing Hua ${ }^{2}$ Xiufeng Liu ${ }^{2}$, Liuqing Yang ${ }^{2}$, Min Yang ${ }^{1}$ \\ ${ }^{1}$ Department of Radiotherapy, The First Affiliated Hospital of Nanjing Medical College, Nanjing 210029, China; ${ }^{2}$ Cancer Centre of People's \\ Liberation Army, Jinling Hospital, Nanjing 210002, China \\ Contributions: (I) Conception and design: F Wang, X Sun, M Yang, S Qin; (II) Administrative support: S Qin; (III) Provision of study materials or \\ patients: F Wang, S Qin, H Hua, X Liu, L Yang; (IV) Collection and assembly of data: F Wang; (V) Data analysis and interpretation: F Wang, X Sun, \\ M Yang, S Qin; (VI) Manuscript writing: All authors; (VII) Final approval of manuscript: All authors. \\ \#These authors contributed equally to this work. \\ Correspondence to: Min Yang. Department of Radiotherapy, The First Affiliated Hospital of Nanjing Medical College, No.300, Guangzhou Road, \\ Nanjing 210029, China. Email: yangmin@jsinm.org; Shukui Qin. Department of Clinical Oncology, Cancer Centre of People’s Liberation Army, \\ Jinling Hospital, No. 34, 34 Biao, Yanggongjing Street, Nanjing 210002, China. Email: qinsk@csco.org.cn.
}

\begin{abstract}
Background: Immune checkpoint inhibitor (ICI)-associated myocarditis is a rare and potentially fatal immune-related adverse event (irAE). The study aimed to observe the occurrence of myocarditis caused by ICIs.

Methods: The clinical manifestations, diagnosis, and treatment of immune myocarditis were explored through retrospective analysis of the detailed data of typical ICI-associated myocarditis from our center and a literature review.

Results: From January 1, 2018, to December 31, 2019, a total of 283 patients were treated with PD-1 or PD-L1 monoclonal antibodies (McAbs) alone or combination therapy at our center. There were 3 cases of ICI-associated myocarditis, of which the incidence rate was $1.06 \%(3 / 283)$; among these cases, 2 were treated with nivolumab alone, and 1 was treated with camrelizumab combined with gemcitabine. One case died on day 56 because of heart and respiratory failure, and the other died on day 34 because of tumor progression. The third case recovered after treatment. The typical clinical manifestations are palpitations, dyspnea, and fatigue. One patient had no clear symptoms. Electrocardiograms (ECG) showed grade 3 of atrioventricular block and frequent ventricular premature contraction in one case, and frequent ventricular and atrial premature contraction in the other case. Most of the cardiac biomarkers decreased or returned to normal after glucocorticoid treatment.
\end{abstract}

Conclusions: ICI-associated myocarditis is a rare adverse event but has a high mortality rate. Early diagnosis of myocarditis and prompt glucocorticoid therapy may be helpful to improve the prognosis.

Keywords: Immune checkpoint inhibitor (ICI); myocarditis; cardiac biomarker; glucocorticoid

Submitted Feb 02, 2020. Accepted for publication Mar 11, 2020.

doi: $10.21037 /$ cco.2020.03.08

View this article at: http://dx.doi.org/10.21037/cco.2020.03.08

\section{Introduction}

In recent years, immune checkpoint inhibitors (ICIs) have emerged as a new type of treatment option drug for malignant tumors. Among them, monoclonal antibodies (McAbs) against programmed cell death protein-1 (PD-1) and its ligand (PD-L1) have been approved for more than 20 types of malignant tumors, including melanoma (1), nonsmall cell lung cancer (2), kidney cancer (3) and urothelial cancer (4). However, these drugs may overstimulate the immune function of the body, resulting in multiple systemic immune-related adverse events (irAEs) including rash, 


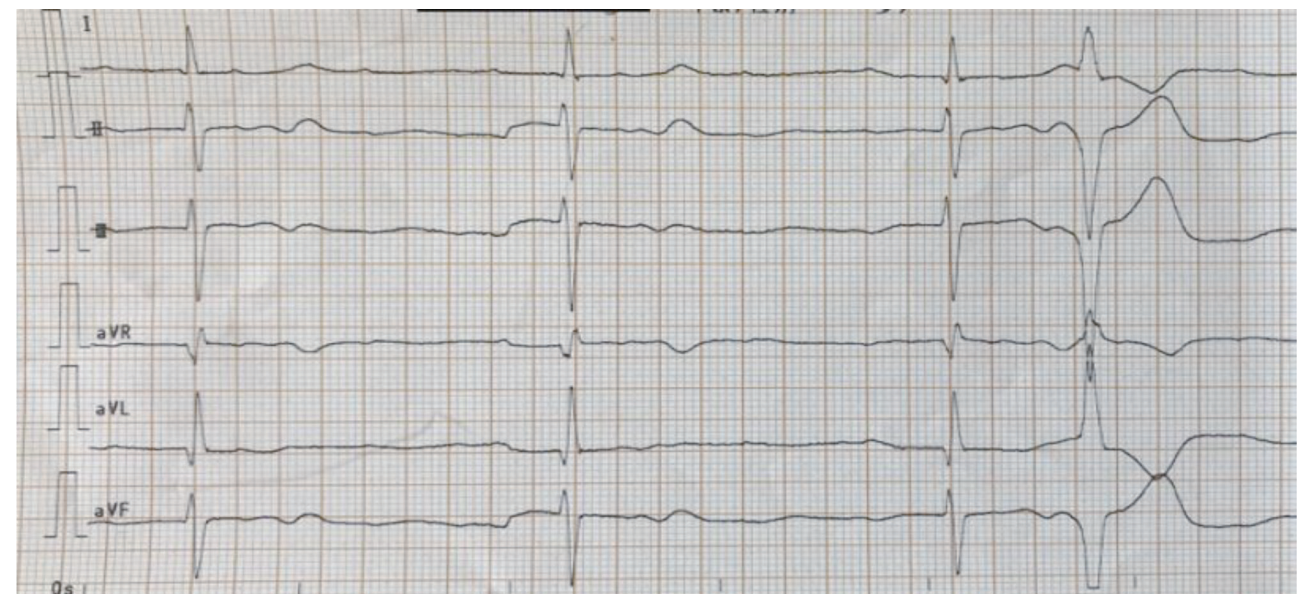

Figure 1 ECG shows grade 3 of atrioventricular block; frequent ventricular premature contraction.

colitis, hepatitis, endocrinopathies, and pneumonitis (5). ICI-associated myocarditis is rare but severe, accounting for a remarkably high mortality rate in clinical practice. With the widespread use of ICIs and the improvement of the cognitive level, the number of reports has gradually increased. In this paper, 3 cases of ICI-associated myocarditis in our center were analyzed, and the literature was carefully reviewed as follows.

\section{Methods}

This study retrospectively analyzed the irAEs caused by PD-1 or PD-L1 McAbs. By analyzing the details of patients with ICI-related myocarditis treated at our center from 2018 to 2019, combined with a literature review, we investigated the clinical presentation, diagnosis, and treatment of immune myocarditis.

\section{Results}

From January 1, 2018, to December 31, 2019, 283 patients at our center were treated with PD-1 or PD-L1 McAbs, including nivolumab, pembrolizumab, camrelizumab, toripalimab, atezolizumab, sintilimab, tislelizumab, and durvalumab. Among these patients, 105 cases were treated with PD-1 or PD-L1 inhibitor only; 178 cases were treated with PD-1 or PD-L1 inhibitor plus chemotherapy, molecular targeted drug or CTLA-4 inhibitor. There were 3 cases of ICI-associated myocarditis, with an incidence of $1.06 \%(3 / 283)$. Two of these cases were treated with nivolumab alone, and the other case was treated with camrelizumab combined with gemcitabine. Myocarditis occurred on the 10th, 13th and 41st days after treatment, respectively. Two patients died (on day 35 and day 56 , respectively), while the third case recovered after treatment. The patients' details are as follows.

Case 1, a 73-year-old woman was diagnosed as metastatic melanoma of the left foot and had a history of hypertension. She was given a dose of nivolumab (PD-1 inhibitor) $100 \mathrm{mg}$ on July 5, 2018. Twelve days later, cardiac biomarker examination showed creatine kinase (CK) 1,349 U/L (normal range, 32-294 U/L), CK-MB $6.2 \mathrm{ng} / \mathrm{mL}$ (normal, 0-3.7 ng/mL), myoglobin (MYO) $883 \mathrm{ng} / \mathrm{mL}$ (normal, 9-96 ng/mL), and troponin I $0.078 \mathrm{ng} / \mathrm{mL}$ (normal, $\leq 0.09 \mathrm{ng} / \mathrm{mL}$ ). The patient's electrocardiogram (ECG) and echocardiography results were almost normal. She had no obvious heart-related symptoms. The second infusion of nivolumab was postponed. The patient was admitted to our hospital due to later palpitations, chest tightness, fatigue, and left eyelid ptosis on July 23, 2018. ECG showed grade 3 atrioventricular block and frequent ventricular premature contraction (Figure 1). CK-MB was increased to $440.4 \mathrm{ng} / \mathrm{mL}$, MYO to $898 \mathrm{ng} / \mathrm{mL}$, and troponin I to $20.935 \mathrm{ng} / \mathrm{mL}$. The patient was diagnosed as ICI-related myocarditis and treated with isoprenaline $1 \mathrm{mg}$ and methylprednisolone $80 \mathrm{mg}(1.2 \mathrm{mg} / \mathrm{kg} / \mathrm{d})$ intravenously. Since the patient had a heart rate of 30-40 times/min, a temporary pacemaker was installed on July 24, 2018, and a permanent pacemaker was installed six days later. After glucocorticoid treatment, CK, CK-MB, troponin, I gradually decreased, but MYO continued to be above $1,000.00 \mathrm{ng} / \mathrm{mL}$. The patient was depressed and fatigue 


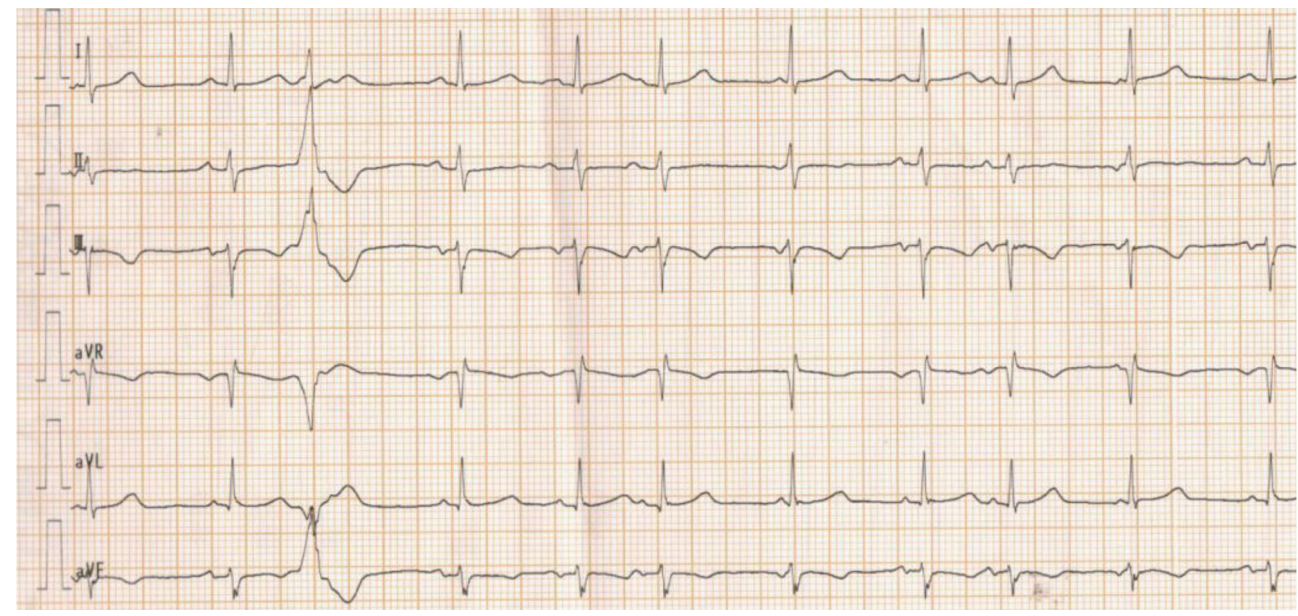

Figure 2 ECG shows frequent atrial and ventricular premature contractions; ST-T segment changes.

aggravated, and she refused any further treatment at the later stage of hospitalization. The patient died of heart and respiratory failure on August 29, 2018.

Case 2, an 80-year-old female patient with a history of hypertension and angina pectoris of coronary heart disease was diagnosed as metastatic renal pelvis urothelial carcinoma. She was treated with nivolumab $200 \mathrm{mg}$ on December 3, 2018. Skin rashes (grade 1) occurred four days later, and fever (grade 2) and vomiting (grade 1) occurred seven days later. Anti-infection treatment was given as a result of respiratory tract infection. On December 12, 2018, sudden dyspnea occurred, and blood oxygen saturation was measured at about $70 \%$. ECG showed frequent atrial and ventricular premature contractions and ST-T segment changes (Figure 2). Biochemical examination showed creatinine $144 \mu \mathrm{mol} / \mathrm{L}$ (normal, 44-97 $\mu \mathrm{mol} / \mathrm{L}$ ). The patient's cardiac biomarkers were: CK 796 U/L, CK-MB $10.8 \mathrm{ng} / \mathrm{mL}, \mathrm{MYO}>1,000 \mathrm{ng} / \mathrm{mL}$, and troponin I $3.9 \mathrm{ng} / \mathrm{mL}$. Arterial blood gas analysis was as follows: $\mathrm{pH} 7.345$, the partial pressure of carbon dioxide: $43.7 \mathrm{mmHg}$; the partial pressure of oxygen: $59.7 \mathrm{mmHg}$; and arterial oxygen saturation: $88.9 \%$. ICI-associated myocarditis was diagnosed accompanied by renal dysfunction and type I respiratory failure. Methylprednisolone $160 \mathrm{mg}(2.5 \mathrm{mg} / \mathrm{kg} / \mathrm{d})$ was administered intravenously, oxygen was given by face mask, and dopamine was given to support blood pressure due to low blood pressure. On December 13, 2018, the patient's CK, CK-MB, and MYO levels were normal; troponin I had decreased to $0.855 \mathrm{ng} / \mathrm{mL}$. Oxygen saturation increased to 90-95\%. On December 30, 2018, the cardiac biomarkers returned to the baseline value but arrhythmia persisted. The course of the disease was accompanied by myasthenia and poor appetite. The patient died on January 6, 2019, due to the rapid progression of the tumor itself.

Case 3, a 56-year-old woman with no previous history of heart disease was diagnosed as metastatic gallbladder adenocarcinoma. Camrelizumab (PD-1 inhibitor made in China) combined with gemcitabine was given for two cycles from July 24, 2019, to August 14, 2019. Forty-one days later, after initiation of the PD-1 inhibitor, the cardiac biomarker examination showed CK 1,084 U/L, CK-MB $10.8 \mathrm{ng} / \mathrm{mL}$, MYO $234 \mathrm{ng} / \mathrm{mL}$, and troponin I $0.081 \mathrm{ng} / \mathrm{mL}$. The patient had no heart-related symptoms, and the ECG and echocardiography were normal. With subclinical immune-mediated myocarditis considered, she was given $4 \mathrm{mg} / \mathrm{kg} / \mathrm{d}$ of methylprednisolone by an intravenous drip of d1-7 and then dose gradually decreased. On the fifth day, the patient's CK, CK-MB, and MYO levels were normal; troponin I was decreased to $0 \mathrm{ng} / \mathrm{mL}$ on the 13 th day. The glucocorticoid was stopped on November 4, 2019. There were no heart-related symptoms at the end of the last follow-up on January 20, 2020, and she received new antitumor treatment.

\section{Discussion}

ICI is a new broad-spectrum anti-tumor drug. The irAEs caused by ICI are mostly grade $1-2$, and the majority can be relieved by discontinuation of drugs or administration of glucocorticoids; however, a small number of patients still experience fatal adverse reactions, such as severe colitis, pneumonia, and hepatitis. Among all irAEs, myocarditis 
accounts for the highest mortality rate (6). In 2013, Voskens et al. (7) reported the first death case of ipilimumab-induced cardiac toxicity. The drugs reported in the literature so far include: ipilimumab $(8,9)$, nivolumab $(10,11)$, pembrolizumab $(12,13)$, and the combination of PD-1 inhibitor and CTLA-4 inhibitor (14,15). Moslehi et al. (16) analyzed 101 cases of reported ICI-associated myocarditis, of which $57 \%$ were treated with PD-1 inhibitors and $27 \%$ were treated with PD-1 inhibitors combined with ipilimumab; and the mortality rate was as high as $46 \%$ (46/101). A retrospective study analyzed the irAEs through the FDA Adverse Events Reports System (FAERS) public dashboard in 2017-2018. A total of 36,848 toxicities of immunotherapies were reported. Out of these, 2,316 (6.3\%) were cardiovascular toxicities, and 816 were ultimately fatal. The most common cardiac complications were as follows: myocarditis (15\%), atrial fibrillation (13\%), pericardial disease including pericardial effusion $(13 \%)$, cardiac failure $(17 \%)$, and coronary artery disease (19\%). The mortality rate of myocarditis was as high as $50 \%(17)$.

The mechanism of immune cardiotoxicity is not clear. Preclinical studies showed that PD-1 and PD-L1 could be expressed in human cardiomyocytes after injury (18). The upregulated T cells of CTLA-4-deficient mice can infiltrate the heart tissue (19). PD-1-deficient mice produce a high titer autoantibody against troponin, which causes dilated cardiomyopathy (20,21). PD-1 and CTLA4-deficient mice may develop fatal myocarditis (22). Lymphocytes in myocardium and tumors showed clonality of TCR, suggesting that heart and tumors can share antigens recognized by the same T-cell clones $(18,23)$. The myocardial biopsies of patients with myocarditis showed a large number of lymphocytic infiltration, usually involving the atrioventricular node and sinoatrial node, with or without myocardial fibrosis. Infiltrating cells within the myocardium and skeletal muscle were positive for the T-cell marker CD3 or the macrophage marker CD68. T-cell infiltrates showed abundant CD4 and CD8 positive $\mathrm{T}$ cells (14).

The incidence of immune myocarditis is less than $1 \%$ (14). However, the actual incidence may be underestimated due to non-specific clinical manifestations and lack of regular cardiac biomarker testing. Myocarditis often occurs in the early stage of ICI treatment, and the incidence and mortality of myocarditis significantly increase with CTLA-4 and PD-1 inhibitor combination therapy. In a retrospective study, $76 \%$ of myocarditis occurred within the six weeks after treatment, with a median of 27 days (5-155 days). The rate of myocarditis caused by PD-1 or PD-L1 inhibitor combined with CTLA-4 inhibitor was significantly higher than that of PD-1 or PD-L1 inhibitor alone $(67 \%$ vs. $36 \% ; \mathrm{P}=0.008$ ) (16). Of 613 fatal toxic events caused by ICIs reported in VigiBase (WHO database) from 2009 to 2018: 333 deaths were related to PD-1/PD-L1 inhibitors, including 27 deaths due to myocarditis, accounting for $8 \%$; and 87 deaths were related to the combination of PD-1/PD-L1 and CTLA-4 inhibitors, including 22 deaths caused by to myocarditis, accounting for $25 \%$ (6). In a retrospective study up to 2018, of 31,321 irAEs, 122 cases were myocarditis $(122 / 31,321,0.39 \%)$, including 84 cases of PD-1/PD-L1 inhibitors, 6 cases of CTLA-4 inhibitors, and 32 cases of PD-1/PD-L1 and CTLA-4 combination therapy; and 95 cases were pericardial diseases, including 74 cases of PD-1/PD-L1 inhibitors, 13 cases of CTLA-4 inhibitors, and 8 cases of combination therapy. The median time of occurrence of myocarditis and pericarditis was 30 days after treatment. The mortality of myocarditis and pericarditis was 50\% (61/122) and 21\% (20/95), respectively. Pericardial diseases were reported more often in patients with lung cancer, while myocarditis was more commonly reported in patients with melanoma (24). A retrospective study from eight centers (25) showed that the incidence of myocarditis was $1.14 \%(11 / 964)$, and the incidence of myocarditis was $0.5 \%, 2.4 \%, 3.3 \%, 2.4 \%$, and 1\% for PD-1 inhibitor, PDL1 inhibitor, CTLA-4 inhibitor, PD-1 combined CTLA4 inhibitor, and PD-L1 combined CTLA-4 inhibitor, respectively. The median time of onset was 34 days (2175 days) after ICI treatment. The age of onset was $65 \pm 13$ years old. Most patients had no history of heart disease, and combination therapy (34\% vs. $2 \% ; \mathrm{P}<0.001)$ and diabetes $(34 \%$ vs. $13 \% ; \mathrm{P}=0.01)$ were more common. Cases 1 and 2 from our center developed myocarditis on the 10 th and 13 th day after the first infusion of PD-1 inhibitors, and case 3 developed myocarditis on the 41st day after 2 cycles of combined PD-1 inhibitor and chemotherapy. Despite active treatment, cases 1 and 2 still died, suggesting that the prognosis of ICI-related myocarditis is extremely poor.

The diagnosis of the cardiac toxicity of ICIs was based on the drug history, clinical manifestations, cardiac biomarkers, ECG, and imaging examination. The clinical presentations of immune-mediated myocarditis vary, from asymptomatic troponin elevation to recurrent chest pain, cardiogenic shock, conduction abnormalities leading to malignant arrhythmias, and sudden death $(8,12,14)$. In an analysis of 30 patients with ICI-related cardiotoxicity, 
it was found that $79 \%$ had decreased left ventricular systolic function, $14 \%$ had Takotsubo syndrome, $30 \%$ had atrial fibrillation, $27 \%$ had ventricular arrhythmia, $17 \%$ had cardiac conduction dysfunction, $23 \%$ had myositisrelated symptoms, $27 \%$ (8/30) died of cardiovascular complications. Cardiovascular mortality was significantly associated with conduction abnormalities $(80 \%$ vs. $16 \%$; $\mathrm{P}=0.003)$ and ipilimumab-nivolumab combination therapy (57\% vs. 17\%; $\mathrm{P}=0.04$ ) (26). Mahmood et al. (25) reported 35 patients with myocarditis and found that the patients could experience chest pain, shortness of breath, dyspnea, in addition to abnormal ECG, increased biomarkers of the heart, cardiac MR myocardial edema, decreased left ventricular ejection fraction, and increased BNP. Cardiac biomarkers usually include troponin (troponin $\mathrm{T}$ or troponin I), CK, and CK-MB. Troponin is most helpful for the diagnosis of ICI-related myocarditis. There was a 4-fold increased risk of major adverse cardiac events (MACE) with troponin $\mathrm{T}$ of $\geq 1.5 \mathrm{ng} / \mathrm{mL}$. However, the diagnosis of myocarditis cannot be determined by the increase of troponin (27). The increase of CK and CK-MB also needs to consider cardiotoxicity, especially myocarditis (14), which is more evident in combination with myositis $(28,29)$. Increased BNP and NT proBNP levels or serum antistriated muscle antibodies also support the diagnosis of ICIs cardiotoxicity (30,31). It was reported that almost all cases of myocarditis had increased troponin (94\%) and abnormal ECG (89\%). ECG showed conduction abnormality, atrial or ventricular arrhythmia. TTE or CMR should be used to examine patients suspected of ICI-associated cardiotoxicity. Cardiac magnetic resonance imaging is a useful diagnostic method in the early stage of the disease, which can show signs of myocarditis. It is worth noting that many patients diagnosed with fulminant ICI-associated myocarditis keep LV function on TTE and CMR $(14,25)$. When ICIs related to myocarditis are clinically suspected, endocardial and myocardial biopsy (EMB) is the gold standard for diagnosis. Histopathological analysis often finds interstitial fibrosis, lymphocytic infiltration, usually involving atrioventricular nodes and sinoatrial nodes, composed of CD8+ T cells and macrophages. B lymphocytes and plasma cells are usually rare $(26,32)$. However, since myocarditis can be characterized by patchy lymphocytic infiltration in the adjacent areas of myocardial necrosis, false-negative pathological cases may also occur.

In case $1, \mathrm{CK}, \mathrm{CK}-\mathrm{MB}$, and MYO increased on the 13th day of PD-1 inhibitor administration. Her troponin I was slightly increased but still in the normal range, and there were no clear symptoms. On the 19th day, the symptoms of palpitation, chest tightness, and fatigue suddenly appeared, and CK, CK-MB, MYO, and troponin I increased significantly. ECG showed grade 3 of atrioventricular block and frequent premature ventricular contraction. Although the patient was treated with glucocorticoid, MYO was higher than $1,000 \mathrm{ng} / \mathrm{mL}$ continuously, and she eventually died on the 56th day. It was suggested that the sensitivity of troponin I was weak when myocarditis was in a subclinical state, while the continuous increase of MYO indicated a poor prognosis. In case 2 , dyspnea occurred suddenly on the 10th day after PD-1 inhibitor administration. CK, CK$\mathrm{MB}, \mathrm{MYO}$, and troponin I were significantly higher than average. The patient also developed other irAEs such as a rash, fever, renal dysfunction, and type 1 respiratory failure. ECG showed frequent atrial and ventricular premature contraction. In case 3, only CK, CK-MB, and MYO were significantly increased. The patient's troponin I was elevated from the baseline, but was also in the normal range and without clinical symptoms. Because myocarditis mostly occurs in the early stage of ICI treatment, it should be paid attention to, and it is necessary to carry out continuous monitoring of cardiac biomarkers and ECG at baseline and within two months after treatment.

The commonly used therapeutic drugs for ICIassociated cardiac toxicity include glucocorticoids and immunosuppressants such as infliximab, antithymocyte globulin (ATG). Glucocorticoids are the first choice of drugs $(33,34)$. Most of them use high dose glucocorticoids, such as methylprednisolone. The first dose is $1-2 \mathrm{mg} / \mathrm{kg}$, which is then gradually reduced. It has been reported that the first dose of methylprednisolone $1,000 \mathrm{mg}$ is related to the lower MACE (25). However, several studies have shown that corticosteroids alone may not be enough to resolve the immune-mediated side effects, even after steroid treatment, and many patients still have malignant arrhythmias and worsening symptoms of heart failure $(8,25)$. A study (35) showed that two patients began to infuse infliximab after a relapse of steroid-refractory myocarditis, showing significant clinical and biochemical improvement, and troponin I decreased to normal. Other studies had shown that infliximab was used in severe steroid-refractory myocarditis $(8,14,30,36)$. One observation showed that two patients responded well to ATG treatment, which could alleviate malignant arrhythmia and cardiogenic shock (35). Other therapeutic drugs include diuretics, $\beta$-blockers, ACEI inhibitors, and antiarrhythmic drugs. Cardiotoxicity should also be managed at different levels and in close cooperation 
with cardiologists. Patients with cardiac toxicity, considering the possibility of fulminant and fatal myocarditis, will not receive ICI treatment again (37). However, it has been reported that if ICIs are used again in patients diagnosed with ICI cardiotoxicity attack, cardiotoxicity does not occur again. In patients with mild cardiotoxicity, the complete reversal of left ventricular systolic dysfunction may be as high as $50 \%$ (26). Therefore, individual treatment should be given based on the treatment effect and risk assessment of patients.

Case 1 was observed in the subclinical period without treatment. After 6 days, the symptoms were fulminant. After glucocorticoid treatment, most of cardiac biomarkers decreased slowly. Permanent pacemaker was used to treat grade 3 of atrioventricular block, but the patient eventually died of heart and respiratory failure. In case 2 , the symptoms were fulminant, and myocarditis was diagnosed in the early stage. After glucocorticoid treatment, the biomarker of heart decreased rapidly, which showed that myocarditis was sensitive to glucocorticoid, but the arrhythmia did not improve significantly. The patient ultimately died of tumor progression. Case 3 was promptly treated with glucocorticoid in the asymptomatic period so that the biomarkers of the heart were rapidly reduced to the baseline level within two weeks, and glucocorticoid was used for eight weeks in total. Up to now, the patient has experienced no cardiac discomfort. Therefore, the rise of cardiac biomarkers may happen earlier than the occurrence of clinical symptoms. For some patients in the subclinical stage, close monitoring of cardiac biomarkers is helpful for early diagnosis. Based on our experience, when $\mathrm{CK}, \mathrm{CK}-\mathrm{MB}$, and MYO reach more than 2.5 times the average value and troponin is significantly higher than the baseline level, subclinical myocarditis should be considered for diagnosis. For subclinical myocarditis, the first dose of methylprednisolone is recommended to be $4 \mathrm{mg} / \mathrm{kg} / \mathrm{d}$, lasting for about one week, before gradually being reduced. After returning to the baseline level, glucocorticoid treatment should continue for about six weeks.

\section{Conclusions}

ICI-associated cardiotoxicity is rare. Among such cases, myocarditis accounts for the highest mortality rate, with most deaths occurring in the early stage after treatment. The clinical manifestations of cardiac toxicity vary. Regular monitoring of cardiac biomarkers is helpful for early diagnosis. The diagnostic criteria of subclinical myocarditis need to be further defined. After the diagnosis of myocarditis, enough glucocorticoids should be given, and a specialized cardiologist should be invited to assist in the diagnosis and treatment of the patient. At the same time as treating myocarditis, we should deal with other immune-related toxicities in time, and prevent and treat glucocorticoid-related side effects such as gastric mucosa injury, hypocalcemia, hypertension, and hyperglycemia. Additionally, we should also manage primary diseases and tumor-related symptoms well to improve the patient's quality of life and prognosis to the greatest extent possible.

\section{Acknowledgments}

Funding: None.

\section{Footnote}

Conflicts of Interest: All authors have completed the ICMJE uniform disclosure form (available at http://dx.doi. org/10.21037/cco.2020.03.08). SQ serves as an unpaid Editor-in-Chief of Chinese Clinical Oncology. The other authors have no conflicts of interest to declare.

Ethical Statement: The authors are accountable for all aspects of the work in ensuring that questions related to the accuracy or integrity of any part of the work are appropriately investigated and resolved. This study was approved by the Ethics Committee of Nanjing Baiyi Hospital (No. 81YY-KYLL-17-11).

Open Access Statement: This is an Open Access article distributed in accordance with the Creative Commons Attribution-NonCommercial-NoDerivs 4.0 International License (CC BY-NC-ND 4.0), which permits the noncommercial replication and distribution of the article with the strict proviso that no changes or edits are made and the original work is properly cited (including links to both the formal publication through the relevant DOI and the license). See: https://creativecommons.org/licenses/by-nc-nd/4.0/.

\section{References}

1. Robert C, Long GV, Brady B, et al. Nivolumab in previously untreated melanoma without BRAF mutation. N Engl J Med 2015;372:320-30.

2. Herbst RS, Baas P, Kim DW, et al. Pembrolizumab versus docetaxel for previously treated, PD-L1-positive, 
advanced non-small-cell lung cancer (KEYNOTE-010): a randomised controlled trial. Lancet 2016;387:1540-50.

3. Motzer RJ, Escudier B, McDermott DF, et al. Nivolumab versus everolimus in advanced renal-cell carcinoma. $\mathrm{N}$ Engl J Med 2015;373:1803-13.

4. Rosenberg JE, Hoffman-Censits J, Powles T, et al. Atezolizumab in patients with locally advanced and metastatic urothelial carcinoma who have progressed following treatment with platinum-based chemotherapy: a single-arm,multicentre,phase 2 trial. Lancet 2016;387:1909-20.

5. Friedman CF, Proverbs-Singh TA, Postow MA. Treatment of the Immune-Related Adverse Effects of Immune Checkpoint Inhibitors: A Review. JAMA Oncol 2016;2:1346-53.

6. Wang DY, Salem JE, Cohen JV, et al. Fatal Toxic Effects Associated With Immune Checkpoint Inhibitors: A Systematic Review and Meta-analysis. JAMA Oncol 2018;4:1721-8.

7. Voskens CJ, Goldinger SM, Loquai C, et al. The price of tumor control: an analysis of rare side effects of antiCTLA-4 therapy in metastatic melanoma from the ipilimumab network. PLoS One 2013;8:e53745.

8. Heinzerling L, Ott PA, Hodi FS, et al. Cardiotoxicity associated with CTLA4 and PD1 blocking immunotherapy. J Immunother Cancer 2016;4:50.

9. Berg DD, Vaduganathan M, Nohria A, et al. Immunerelated fulminant myocarditis in a patient receiving ipilimumab therapy for relapsed chronic myelomonocytic leukaemia. Eur J Heart Fail 2017;19:682-5.

10. Behling J, Kaes J, Münzel T, et al. New-onset third-degree atrioventricular block because of autoimmune-induced myositis under treatment with anti-programmed cell death-1 (nivolumab) for metastatic melanoma. Melanoma Res 2017;27:155-8.

11. Matson DR, Accola MA, Rehrauer WM, et al. Fatal Myocarditis Following Treatment with the PD-1 Inhibitor Nivolumab. J Forensic Sci 2018;63:954-7.

12. Läubli H, Balmelli C, Bossard M, et al. Acute heart failure due to autoimmune myocarditis under pembrolizumab treatment for metastatic melanoma. J Immunother Cancer 2015;3:11.

13. Zimmer L, Goldinger SM, Hofmann L, et al. Neurological, respiratory, musculoskeletal, cardiac and ocular side-effects of anti-PD-1 therapy. Eur J Cancer 2016;60:210-25.

14. Johnson DB, Balko JM, Compton ML, et al. Fulminant myocarditis with combination immune checkpoint blockade. N Engl J Med 2016;375:1749-55.

15. Mahmood SS, Chen CL, Shapnik N, et al. Myocarditis with tremelimumab plus durvalumab combination therapy for endometrial cancer: A case report. Gynecol Oncol Rep 2018;25:74-7.

16. Moslehi JJ, Salem JE, Sosman JA, et al. Increased reporting of fatal immune checkpoint inhibitor-associated myocarditis. Lancet 2018;391:933.

17. Master SR, Robinson A, Mills GM, et al. Cardiovascular complications of immune checkpoint inhibitor therapy. J Clin Oncol 2019;37:Abstract 2568.

18. Varricchi G, Galdiero MR, Tocchetti CG. Cardiac Toxicity of Immune Checkpoint Inhibitors: Cardio-Oncology Meets Immunology. Circulation 2017;136:1989-92.

19. Waterhouse P, Penninger JM, Timms E, et al. Lymphoproliferative disorders with early lethality in mice deficient in Ctla-4. Science 1995;270:985-8.

20. Nishimura H, Okazaki T, Tanaka Y, et al. Autoimmune dilated cardiomyopathy in PD-1 receptor-deficient mice. Science 2001;291:319-22.

21. Okazaki T, Tanaka Y, Nishio R, et al. Autoantibodies against cardiac troponin $\mathrm{I}$ are responsible for dilated cardiomyopathy in PD-1-deficient mice. Nat Med 2003;9:1477-83.

22. Wang J, Okazaki IM, Yoshida T, et al. PD-1 deficiency results in the development of fatal myocarditis in MRL mice. Int Immunol 2010;22:443-52.

23. Tocchetti CG, Galdiero MR, Varricchi G. Cardiac Toxicity in Patients Treated With Immune Checkpoint Inhibitors: It Is Now Time for Cardio-Immuno-Oncology. J Am Coll Cardiol 2018;71:1765-7.

24. Salem JE, Manouchehri A, Moey M, et al. Cardiovascular toxicities associated with immune checkpoint inhibitors: an observational, retrospective, pharmacovigilance study. Lancet Oncol 2018;19:1579-89.

25. Mahmood SS, Fradley MG, Cohen JV, et al. Myocarditis in Patients Treated With Immune Checkpoint Inhibitors. J Am Coll Cardiol 2018;71:1755-64.

26. Escudier M, Cautela J, Malissen N, et al. Clinical Features, Management, and Outcomes of Immune Checkpoint Inhibitor-Related Cardiotoxicity. Circulation 2017;136:2085-7.

27. Sarocchi M, Grossi F, Arboscello E, et al. Serial Troponin for Early Detection of Nivolumab Cardiotoxicity in Advanced Non-Small Cell Lung Cancer Patients. Oncologist 2018;23:936-42.

28. Touat M, Maisonobe T, Knauss S, et al. Immune checkpoint inhibitor-related myositis and myocarditis in 
patients with cancer. Neurology 2018;91:e985-94.

29. Suzuki S, Ishikawa N, Konoeda F, et al. Nivolumab-related myasthenia gravis with myositis and myocarditis in Japan. Neurology 2017;89:1127-34.

30. Frigeri M, Meyer P, Banfi C, et al. Immune Checkpoint Inhibitor-Associated Myocarditis: A New Challenge for Cardiologists. Can J Cardiol 2018;34:92.e1-3.

31. Norwood TG, Westbrook BC, Johnson DB, et al. Smoldering myocarditis following immune checkpoint blockade. J Immunother Cancer 2017;5:91.

32. Koelzer VH, Rothschild SI, Zihler D, et al. Systemic inflammation in a melanoma patient treated with immune checkpoint inhibitors-an autopsy study. J Immunother Cancer 2016;4:13.

33. Haanen JBAG, Carbonnel F, Robert C, et al. Management of toxicities from immunotherapy: ESMO Clinical

Cite this article as: Wang F, Sun X, Qin S, Hua H, Liu X, Yang L, Yang M. A retrospective study of immune checkpoint inhibitor-associated myocarditis in a single center in China. Chin Clin Oncol 2020;9(2):16. doi: 10.21037/cco.2020.03.08
Practice Guidelines for diagnosis, treatment and followup. Ann Oncol 2017;28:iv119-42.

34. Heinzerling L, Goldinger SM. A review of serious adverse effects under treatment with checkpoint inhibitors. Curr Opin Oncol 2017;29:136-44.

35. Agrawal N, Khunger A, Vachhani P, et al. Cardiac Toxicity Associated with Immune Checkpoint Inhibitors: Case Series and Review of the Literature. Case Rep Oncol 2019;12:260-76.

36. Tay RY, Blackley E, McLean C, et al. Successful use of equine anti-thymocyte globulin (ATGAM) for fulminant myocarditis secondary to nivolumab therapy. Br J Cancer 2017;117:921-4

37. Champiat S, Lambotte O, Barreau E, et al. Management of immune checkpoint blockade dysimmune toxicities: a collaborative position paper. Ann Oncol 2016;27:559-74. 